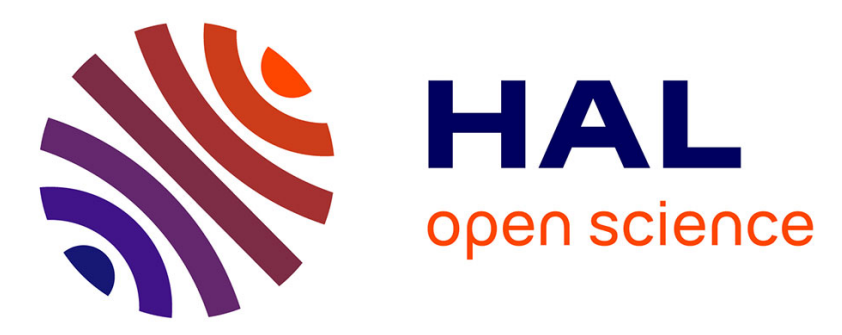

\title{
A reliable and energy-efficient leader election algorithm for Wireless Body Area Networks
}

Rongrong Zhang, Hassine Moungla, Mehaoua Ahmed

\section{To cite this version:}

Rongrong Zhang, Hassine Moungla, Mehaoua Ahmed. A reliable and energy-efficient leader election algorithm for Wireless Body Area Networks. IEEE International Conference on Communications (ICC2015), Jun 2015, London, United Kingdom. pp.530-535, 10.1109/ICC.2015.7248376 . hal-01270447

\section{HAL Id: hal-01270447 \\ https://hal.science/hal-01270447}

Submitted on 17 Feb 2016

HAL is a multi-disciplinary open access archive for the deposit and dissemination of scientific research documents, whether they are published or not. The documents may come from teaching and research institutions in France or abroad, or from public or private research centers.
L'archive ouverte pluridisciplinaire HAL, est destinée au dépôt et à la diffusion de documents scientifiques de niveau recherche, publiés ou non, émanant des établissements d'enseignement et de recherche français ou étrangers, des laboratoires publics ou privés. 


\title{
A Reliable and Energy-Efficient Leader Election Algorithm for Wireless Body Area Networks
}

\author{
Rongrong Zhang, Hassine Moungla, Ahmed Mehaoua \\ LIPADE, University of Paris Descartes, Sorbonne Paris Cité 45 rue des saints pères, 75006, Paris, France \\ Email: \{rongrong.zhang; hassine.moungla; ahmed.mehaoua\}@parisdescartes.fr
}

\begin{abstract}
Wireless Body Area Networks (WBANs) which offer a variety of promising applications in the areas of medical and consumer electronics have been paid lots of attention. However, very limited work has been done on the network reliability combined with energy conservation in spite of their fundamental importance. In order to bridge this gap, we devote this paper to developing a reliable and energy-efficient leader election (REELE) algorithm for WBANs. To this end, technically, we first partition a WBAN into regions and build the reliability and energy consumption models. By the reliability analysis, we then propose a novel communication strategy for nodes and further derive the total energy consumption of a region. With the reliability and residual energy of a node and total energy consumption considered jointly, REELE algorithm can considerably enhance reliability and conserve energy. Extensive simulation results demonstrate the effectiveness and the efficiency of REELE in terms of longer network lifetime, better energy characteristics as well as higher reliability.
\end{abstract}

\section{INTRODUCTION}

Wireless Body Area Networks (WBANs) are the emerging networks with several lightweight sensors to monitor, manage and transmit the real time physiological parameters [1]. Due to the highly extensive potential applications, many researchers pay their attention to study the performance of WBANs. On account of the low power level and scare battery capacity, the network lifetime is one of the most critical performance metrics for WBANs. On the other hand, two-hop cooperative communication assisted by a relay node has drawn much attention in WBANs currently which can achieve a diversity gain that enhances the link reliability. However, how to conserve energy and guarantee the reliable communication is still an open challenge for WBANs.

\section{A. Related work}

For the primary importance of energy conservation on network longevity, considerable researches have been conducted in the past decade [2-4]. In [2], the authors evaluated the network performance in different topologies. On comparison, it is concluded that a cluster-based protocol is more energy efficient in terms of the energy consumption than other graphbased protocols. In [3], the authors presented a Low-Energy Adaptive Cluster Hierarchy (LEACH) protocol which requires that each node takes its turn as cluster head periodically to balance the energy consumption and to avoid the close nodes die earlier. A more profound study on Hybrid Energy-Efficient Distributed (HEED) protocol was performed in [4], which allows the node with more residual energy a bigger probability to become cluster head. And the results showed that HEED protocol is effective in prolonging the network lifetime.

On the other hand, the reliability is a stringent requirement on medical applications closely related to human's health. To the best of our knowledge, there is only limited work on the network reliability for WBANs [5-9]. Due to the high path loss derived in [5] on human body, it has been justified that direct communication with the sink is inefficient and unreliable for far nodes [6]. In [7], authors analyzed the outage probability performance in three transmission schemes, i.e., direct transmission, single-relay cooperative and multirelay cooperative, which demonstrates that the cooperative communication can help to reduce the outage probability and to improve the energy efficiency for WBANs. Moreover, the packet error rate probability and the related outage probability of each on-body link were investigated in [8]. It is observed that cooperative transmission scheme achieves a performance enhancement in terms of packet error rate versus transmitted power. Furthermore, two-hop cooperative communication was adopted in IEEE 802.15.6 standard [9] as an option to overcome significantly the path loss in WBANs.

Motivated by the aforementioned existing analysis, we argue that a novel study based on cluster topology and cooperative communication is called for in order to improve the network reliability and prolong the network lifetime for WBANs.

\section{B. Summary of Contributions}

In this paper, different from the previous work, we propose a Reliable and Energy-Efficient Leader Election (REELE) algorithm for WBANs. The main contributions are articulated as follows:

- Firstly, we partition a WBAN into regions to manage sensors efficiently and introduce the network model.

- Secondly, we model the reliability as the probability of successful communication and present a reliable and energy-efficient communication strategy. Furthermore, the total energy consumption model is formulated.

- Thirdly, we propose a distributed algorithm by jointly considering reliability, residual energy and total energy consumption to select the optimal leader in each region and analyze its complexity.

- Finally, we comprehensively evaluate REELE. The simulation results show REELE has better performance in terms of network lifetime, energy conservation as well as the reliability compared with existing solutions. 


\section{Paper Organization}

The remainder of this paper is organized as follows. Section II introduces the network configuration and network model. In section III, we analyze the reliability and energy consumption model and propose an effective communication strategy. In Section IV, we formally develop a distributed leader election algorithm and compute its complexity. Then, the simulation results on the performance of the proposed algorithm are presented in Section V. Finally, we conclude our paper in section VI.

\section{PREliminaries}

In this section, we first describe the network configuration and introduce the network model which will be used through the rest of this paper subsequently.

\section{A. Network Configuration}

Due to the unique properties of WBANs with distinct channel characteristics and very small scale, the existing results on energy conservation and reliability enhancement may be inadequate if they are applied to WBANs directly. Thus, we make the following improvement on network configuration:

- Firstly, we partition a WBAN into regions as the same definition as the cluster. The idea is derived accordingly from the results that the cluster-based topology is proved more energy-efficient than single-hop, tree-based and line-based topologies in [2]. Moreover, the native symmetry of the human body also supports for this idea.

- Secondly, one and only one sensor in each region can be elected to be the leader like the cluster-head to manage and schedule other sensors effectively. Note that the leader is the relay in cooperative communication to help others to transfer their packets to the sink.

- Thirdly, due to high pass loss around the human body, it has been justified that nodes in the same side of the body can communicate more reliably with each other than with nodes in the other side [6]. This observation prompts us that communication should happen between the nodes in the same side of the body, which also verifies the feasibility of the proposed partitioning idea. Therefore, sensors are deployed in different regions based on individual functions. And the sink is located in the center of the body to collect all the information attained by the sensors and send them to the personal server. We illustrate a realistic emplacement for the sensors of a WBAN in Fig. 1.

Accordingly, based on the region topology and cooperative communication, we develop a reliable and energy-efficient leader election algorithm suitable for WBANs in this paper.

\section{B. Network model}

In this paper, we consider a general WBAN system model with $I$ limited energy sensors and one unlimited energy sink node. Based on the above configuration, we partition a WBAN into $M$ logical regions. For convenient distinction, each sensor is assigned a region identification number $(R I D)$ and a node identification number (NID), respectively. Definitely, the region set in a WBAN is $R=\left\{r_{1}, \ldots, r_{m}, \ldots, r_{M}\right\}$ where $r_{m}$ represents the $m$-th region. Correspondingly, the node set is $N=\left\{n_{1}, \ldots, n_{i}, \ldots, n_{I}\right\}$ where the $i$-th sensor is described as $n_{i}$. Consequently, we can use the dual $\left\langle r_{m}, n_{i}>\right.$ to uniquely indicate the $i$-th sensor in the $m$-th region. Note that in this paper, we use the terms of "node" and "sensor" interchangeably to refer to the same WBAN entity.

Besides, we make the following reasonable assumptions about the nodes and the network model:

- All nodes are of the same sensing and computation capability and always have packets to send during their active periods;

- All nodes are stationary after the deployment. And they know their coordinates and real-time residual energy;

- The leader must communicate with the sink directly, while the other sensors can adopt direct communication or cooperative communication adaptively.

\section{RELIABILITy AND ENERgy CONSUMPTION ANALYSIS}

In this section, we first model the reliability for WBANs and propose an effective communication strategy. Subsequently, the total energy consumption model is detailed.

\section{A. Reliability model}

In order to develop the reliability in WBANs, we firstly need to model the path loss between the transmitting and receiving antennas as a function of the distance $d$. In our analysis, we use the following semi-empirical formula which is expressed in decibel and based on the Friis formula [5]

$$
P L_{d B}(d)=P L_{0, d B}+10 \cdot n \cdot \log \left(d / d_{0}\right),
$$

where $P_{0, d B}$ is the path loss in $\mathrm{dB}$ at a reference distance $d_{0}$ (10 $\mathrm{cm}$ in this paper) and $n$ is the path loss exponent which equals 2 in free space.

In practice, there exists shadowing fading defined as the variation of the local mean around the path loss. To guarantee the reliable communication, it is essential to account for this factor. Thereby, the total path loss becomes a random variable defined as

$$
P L=P L_{d B}+P L_{s},
$$

where $P L_{d B}$ is the value predicted by the path loss model as equation (1), and the shadowing component $P L_{s}$ is represented by a zero-mean Gaussian random variable $X_{\sigma, d B}$ with standard deviation $\sigma$ in $\mathrm{dB}$.

Thus the signal strength $P_{r, d B}^{j}$ at a receiver $n_{j}$ from a transmitter $n_{i}$ with transmitting power $P_{s, d B}^{i}$ over a distance $d_{i j}$ can be computed as

$$
P_{r, d B}^{j}\left(d_{i j}\right)=P_{s, d B}^{i}-P L_{d B}\left(d_{i j}\right)-X_{\sigma, d B}
$$

In this paper, we evaluate the reliability from the perspective of the received signal strength at the receiver as done in wireless communication. If the signal strength $P_{r, d B}^{j}$ is higher than a certain threshold $P_{t h}$, the received packet can be acknowledged. Conversely, the link is interrupted and the unreliable communication will happen. As a result, the reliability can be 
modeled as the probability $p\left(d_{i j}\right)$ of sucessful communication between two nodes $n_{i}$ and $n_{j}$, which is formulated as follows:

$$
\begin{aligned}
p\left(d_{i j}\right) & =\operatorname{Pr}\left[P_{r, d B}^{j}\left(d_{i j}\right)>P_{t h}\right] \\
& =\operatorname{Pr}\left[X_{\sigma, d B}+\mu\left(d_{i j}\right)<0\right],
\end{aligned}
$$

where the left part of the second inequality can be seen as a normal distribution with the standard deviation $\sigma$ and the mean $\mu\left(d_{i j}\right)$ shown as follows:

$$
\mu\left(d_{i j}\right)=-P_{s, d B}^{i}+P L_{0, d B}+10 n \log _{10}\left(d_{i j} / d_{0}\right)+P_{t h} .
$$

Consequently, the probability in (4) can be rewritten as:

$$
\begin{aligned}
p\left(d_{i j}\right) & =\frac{1}{\sqrt{2 \pi} \sigma} \int_{-\propto}^{0} \exp \left[-\frac{\left(t-\mu\left(d_{i j}\right)\right)^{2}}{2 \sigma^{2}}\right] d t \\
& =\frac{1}{2}-\frac{1}{2} \operatorname{erf}\left(\frac{\mu\left(d_{i j}\right)}{\sqrt{2 \pi} \sigma}\right)
\end{aligned}
$$

where $\operatorname{erf}()$ is the standard cumulative error function. It is clear that the reliable communication probability also depends on transmitting power $P_{s, d B}^{i}$ and receiving threshold $P_{t h}$. In IEEE 802.15.6-WBANs standard [9], the former can be set from $-25 \mathrm{dBm}$ to $0 \mathrm{dBm}$. And the later is defined by the parameters of the receiver. For instance, if the noise floor is $-90 \mathrm{dBm}$ and the required signal-to-noise ratio is at least 20 $\mathrm{dB}, P_{t h}$ can be set as $-70 \mathrm{dBm}$.

\section{B. Reliable and energy-efficient communication strategy}

Although cooperative communication scheme has been proved more energy efficient in many cases, it is not applicable to all nodes specially for the small scale WBANs. Moreover, the network reliability is overlooked in many studies. Therefore, by considering energy efficiency and network reliability jointly we propose a novel communication strategy which is applicable to WBANs.

Here, we detail the novel communication strategy. Once the only one leader is elected, correspondingly the other nodes are the normal members in each region. As an assumption, the leader must adopt direct communication with the sink. If a node $n_{k}$ is elected as the leader, a normal node $n_{i}$ will determine whether or not to implement cooperative communication by the following rules: (1) It firstly judges whether its distance to the sink $d_{i, s}$ is larger than that between the leader and the sink $d_{k, s} ;(2)$ and it compares the reliability probability $p\left(d_{i, k}\right)$ - $p\left(d_{k, s}\right)$ of the cooperative communication with that $p\left(d_{i, s}\right)$ of the direct communication. If the inequalities $d_{i, s}>d_{k, s}$ and $p\left(d_{i, k}\right) \cdot p\left(d_{k, s}\right)>p\left(d_{i, s}\right)$ hold, the node will choose cooperative communication. Otherwise, the direct communication is adopted.

The reason for the design is two-fold. For the far nodes, the direct communication with the sink requires a large transmission energy, which quickly drains the battery and shorten the network lifetime. Moreover, the large distance also results in the increase of outage probability. Therefore, cooperative communication is necessary to reduce the energy dissipation and to enhance the reliability of the far nodes. However, for the nodes close to the sink, it is obvious that more energy of both nodes and the leader will be consumed if the leader is farther from the sink. In other words, cooperative communication is not beneficial for the short distance communication.
Besides, the reliability of the source-leader-sink link will be deteriorated instead of improvement in this case. Thus, the direct communication is preferable conversely.

Consequently, when direct communication is preferred by the close nodes, the leader is relieved of its relaying burden. While cooperative communication can offer more advantage in alleviating the long distance transmissions and improving the reliability for the far nodes. Thus adaptively adjusting communication mode can significantly boost network performance.

\section{Total energy consumption model}

In this subsection, we compute the total energy consumption of a region using the effective communication strategy.

In WBANs, communication between nodes and the sink occurs along the surface of the human body. Thus, we exploit the energy consumption model [10] for transmitting and receiving $l$-bits data over distance $d$, shown as follows:

$$
\begin{cases}E_{T x}(l, d, n) & =l E_{\text {Txelec }}+l \epsilon_{a m p}(n) d^{n}, \\ E_{R x}(l) & =l E_{\text {Rxelec }},\end{cases}
$$

where $E_{\text {Txelec }}$ and $E_{R x e l e c}$ are the energy dissipated by the radio to run the circuitry for the transmitter and the receiver respectively, and $\epsilon_{a m p}$ is the energy for the transmission amplifier. $d$ is the distance between the transmitter and the receiver, and $n$ represents the path loss coefficient.

As mentioned in section II, the leader aggregates the packets original from the normal nodes into one single length-fixed packet and will transform it to the sink, so the leader will consume extra $E_{D A}$ energy for the data aggregation. Note that we focus on the energy consumption for communication between nodes and ignore that for sensing and computation.

Before calculating the total energy consumption, we first introduce the following notations. Let node $n_{k}$ be the leader in the $m$-th region. Define an indication variable as follows:

$$
R_{k, m}= \begin{cases}1 & \text { if } n_{k} \text { is the leader in region } r_{m} \\ 0 & \text { otherwise }\end{cases}
$$

which is subjected to $\sum_{m=1}^{M} \sum_{k=1}^{N} R_{k, m}=M$.

As for normal nodes, they employ the proposed reliable and energy-efficient communication strategy. Let $D_{i, m}$ indicate whether node $n_{i}$ chooses the direct communication

$$
D_{i, m}= \begin{cases}1 & \text { if } n_{i} \text { selects direct commun. and } n_{i} \text { is } \\ \text { in the } m \text {-th region, } i \in N, i \neq k, & \text { otherwise. }\end{cases}
$$

Correspondingly, a variable indicating whether node $n_{i}$ chooses the cooperative communication can be described as

$$
C_{i, m}= \begin{cases}1 & \text { if } n_{i} \text { selects cooperative commun. and } \\ & n_{i} \text { is in the } m \text {-th region, } i \in N, i \neq k, \\ 0 & \text { otherwise. }\end{cases}
$$

Thus, the sum of nodes choosing the cooperative communication in the $m$-th region can be expressed as $B_{m}=\sum_{i=1}^{N} C_{i, m}$.

Given the above notations and energy consumption model, the total energy consumption of region $r_{m}$ can be separated into four parts: 1) energy consumption of direct transmission from the normal nodes to the sink, $\left.\sum_{i \in N, i \neq k} D_{i, m} \cdot E_{T x} ; 2\right)$ 
energy consumption of cooperative transmission from the normal nodes to the leader, $\left.\sum_{i \in N, i \neq k} C_{i, m} \cdot E_{T x} ; 3\right)$ energy consumption of the leader used to receive and aggregate the packets from other nodes, $\sum_{k \in N} R_{k, m}\left(E_{R x} \cdot B_{m}+E_{D A}\right)$; 4) energy consumption of transmission from the leader to the sink, $\sum_{k \in N} R_{k, m} \cdot E_{T x}$. As a result, the total energy consumption can be derived as follows:

$$
\begin{aligned}
E_{\text {total }}= & \sum_{i \in N, i \neq k} D_{i, m}\left(l \cdot E_{\text {Txelec }}+l \cdot \varepsilon_{\text {amp }} d_{i, s}^{n}\right)+ \\
& \sum_{i \in N, i \neq k} C_{i, m}\left(l \cdot E_{\text {Txelec }}+l \cdot \varepsilon_{a m p} d_{i, k}^{n}\right)+ \\
& \sum_{k \in N} R_{k, m}\left(l \cdot E_{\text {Rxelec }} \cdot B_{m}+E_{D A}\right)+ \\
& \sum_{k \in N} R_{k, m}\left(l \cdot E_{\text {Txelec }}+l \cdot \varepsilon_{a m p} d_{k, s}^{n}\right)
\end{aligned}
$$

\section{LEAder Election Algorithm}

In this section, we present REELE algorithm for WBANs to elect the region leader based on the reliability and energy consumption analysis and further analyze its complexity.

\section{A. Algorithm design}

The goal of REELE algorithm is to select a best leader in one region to reduce the energy consumption and improve the reliability. On account of the scarce battery capacity and the importance of reliability, REELE primarily screens nodes based on the residual energy and reliability. Then the total energy consumption as the third condition is considered to prolong network lifetime. A formal description is shown in the Algorithm 1 for an arbitrary node $n_{i}$.

In REELE algorithm, we assume that nodes in the same region can receive all control messages from other nodes and know their locations by an ideal neighbor discovery process which is not our focus. Before executing the algorithm, each node initially establishes and updates an information table consisting of its RID, NID, location, initial energy and residual energy. Then, it contends for a tentative leader with the probability $p_{i}(t)$ at time $t$, defined as:

$$
p_{i}(t)=\frac{E_{i}(t)}{E_{0}}
$$

where $E_{i}(t)$ is residual energy and $E_{0}$ is the initial energy. Note that $p_{i}(t)$ decreases as the energy is diminished gradually. Thus, to reduce the probability of no tentative leaders and guarantee the network reliability, we add two constraint conditions on $p_{i}(t)$ and the probability $p\left(d_{i, s}\right)$ of reliable communication between node $n_{i}$ and the sink $s$, respectively. Definitely, $p_{i}(t)>p_{\min }$ and $p\left(d_{i, s}\right)>p_{t h}$ where $p_{\min }$ (e.g., $1 / N)$ and $p_{t h}$ are two thresholds.

Once the expected tentative leaders are elected, they will firstly compute $E_{\text {total }}$ by assuming it is the leader and broadcast a COMPETE_MSG message including its RID, NID and $E_{\text {total }}\left(n_{i}\right)$ to compete the final leader. In order to select the optimal final leader, each tentative leader also maintains a set $S_{n i}$ of its competitors in the same region and compares its total energy consumption $E_{\text {total }}$ with its competitors. If $n_{i}$ finds its $E_{\text {total }}$ the lowest, it will successfully be the final leader and broadcast a LEADER_MSG message including its $R I D, N I D, E_{\text {total }}\left(n_{i}\right), d_{i, s}$ and $p\left(d_{i, s}\right)$. Otherwise, it will give up the competition immediately and inform other nodes by a QUIT_MSG message.

Finally, if node $n_{k}$ becomes the final leader, the normal nodes will choose the communication mode according to the reliable and energy-efficient communication strategy. Nevertheless, if there doesn't exist the final leader, it will return to the initialization and reselect the leader.

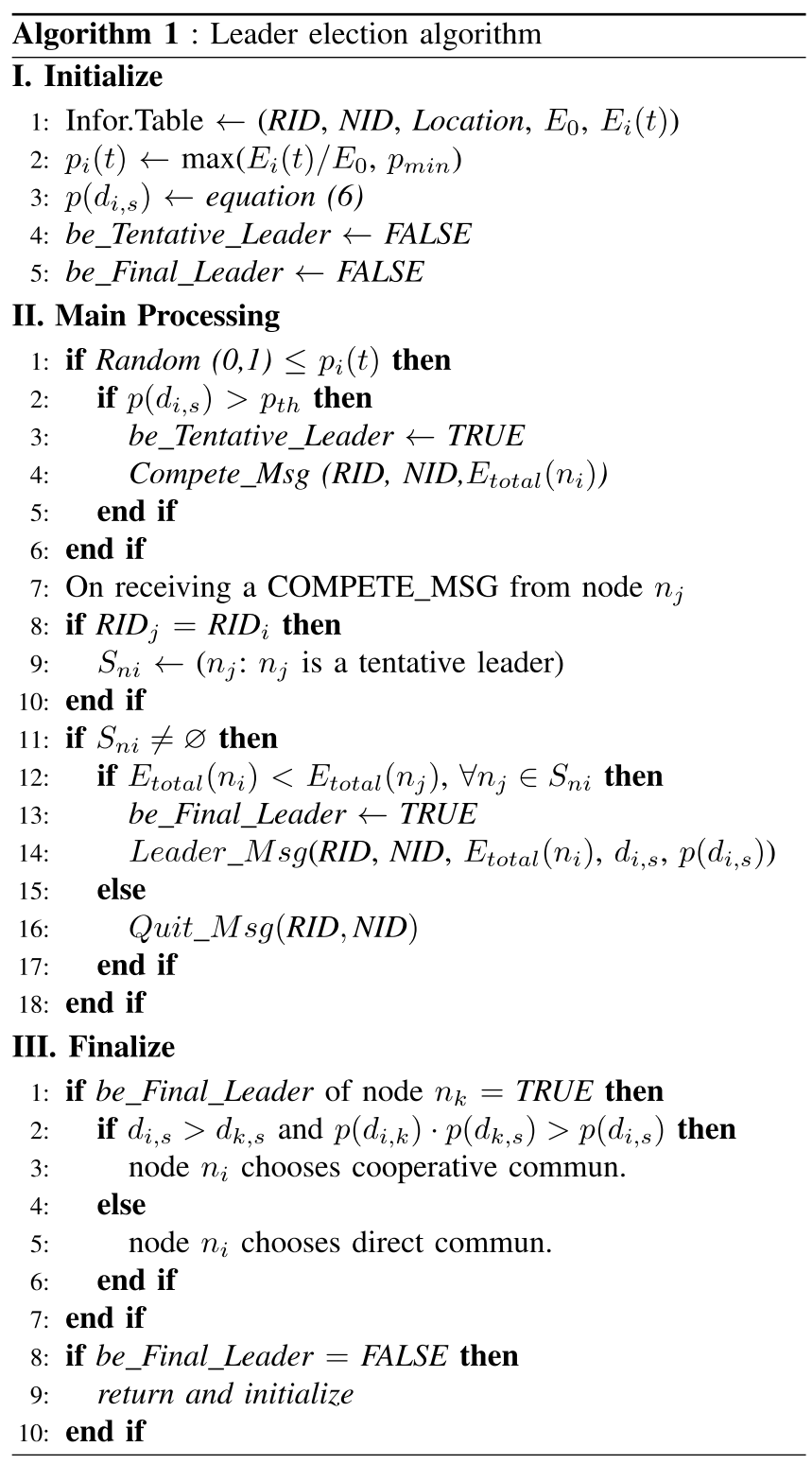

\section{B. Algorithm analysis}

The leader election process in REELE is message driven, thus we discuss its message exchange complexity below.

Lemma 1. REELE algorithm has a worst-case message exchange complexity of $O(I)$ in the network, where $I$ is the number of nodes. 
Proof: During the execution of REELE, there are at most $I \cdot p_{i}(t)$ tentative leaders who broadcast the COMPETE_MSG per round. Then, each of them either broadcasts a LEADER_MSG to act as the final leader or a QUIT_MSG to be a normal node. For only one final leader is elected in one region, there will be $M$ LEADER_MSGs and $\left(I \cdot p_{i}(t)-M\right)$ QUIT_MSGs are transmitted. After the final leader has been elected, each normal node will send a short control message to the leader to confirm their communication modes. Thus, the messages add up to $2 I p_{i}(t)+(I-M)$ per round. Since $p_{i}(t)<1$, the total asymptotic order of the control overhead is $O(I)$.

Remark. The number of nodes in a WBAN is extremely limited for the network characteristics [1]. Consequently, Lemma 1 verifies the message overhead of Algorithm 1 is very small.

\section{Performance Evaluation}

In this section, we evaluate the performance of the proposed REELE algorithm and compare it with two algorithms: LEACH-A and HEED-A. Since there does not exist a study on cluster-based leader election scheme in WBANs, in order to make a fair comparison, LEACH-Analogous and HEEDAnalogous are derived from the most classical energy-efficient protocols of LEACH [3] and HEED [4] for Wireless Sensor Networks (WSNs), respectively. Without loss of generality, an ideal MAC layer and error-free links are assumed.

\section{A. Performance metrics}

In the simulation, we assess the performance of REELE in terms of:

- Network lifetime: Lifetime is the vital criterion for evaluating the performance of WBANs, which is defined as the time until the first node dies in this paper;

- Energy characteristics: To verify the effectiveness and efficiency of REELE on the energy conservation, the average residual energy and the energy efficiency are evaluated. In addition, the average energy consumption of the leader is also assessed.

- Reliability: To justify the improvement in the reliability of REELE, the reliability of each node is investigated.

\section{B. Simulation settings}

Following the analysis in Section II, in the simulation, we partition a WBAN into 4 regions and deploy 13 sensors on human body [1] as shown in Fig. 1. The simulation parameters are detailed in Table I. Moreover, every simulation result is given by the average of 100 independent experiments.

TABLE I

Simulation SETtings

\begin{tabular}{|l|l|}
\hline Parameter & Setting \\
\hline One region coverage & $(0,0) \sim(50,100)$ \\
Initial energy & $0.1 \mathrm{~J}$ \\
$E_{\text {Txelec }}, E_{\text {Rxelec }}$ & $16.7 \mathrm{~nJ} / \mathrm{bit}, 36.1 \mathrm{~nJ} / \mathrm{bit}$ \\
$\epsilon_{\text {amp }}(n)$ & $1.97 \mathrm{~nJ} /\left(\mathrm{bit} \cdot \mathrm{m}^{n}\right),(n=3.11)$ \\
$E_{D A}$ & $5 \mathrm{~nJ} / \mathrm{bit} / \mathrm{signal}$ \\
Data packet size & $4,000 \mathrm{bits}$ \\
ShadowingStd & $\sigma=6.2$ \\
\hline
\end{tabular}

\section{Simulation results and analysis}

1) Network lifetime: Fig. 2 illustrates the network lifetime as the initial energy varies. It is shown that REELE algorithm dramatically improves the lifetime over LEACH-A and HEEDA. In REELE, only the node of the more residual energy and less total energy consumption is elected as the leader so that it not only balances the burden of the leader but also reduces the total energy depletion. Differently, LEACH-A requires each node taking its turn to be the leader, while HEED-A only considers residual energy as main criterion to elect the leader.

2) Energy characteristic: We now evaluate average residual energy and energy efficiency defined as the ratio of total energy consumption to total received bits. Fig. 3 and Fig. 4 manifest that REELE achieves the most residual energy and the lowest energy efficiency. Note that the lower the energy efficiency is, the less energy is expended by transmitting per bit information.

Both LEACH-A and HEED-A overlook the case that a far node also can win as the leader. In this case, it will dissipate more considerable energy to transmit the packets to the sink over a longer distance, which leads to the vast decrease of the residual energy. Thoughtfully, the constraints both on the competitive probability and reliability are used in REELE, dropping the probability of a far node to be the leader. Moreover, the proposed reliable and energy-efficient communication strategy relieves the burden of the leader and far nodes. Consequently, it can achieves more residual energy.

The definition of the energy efficiency implies that under the same amount of the traffic per round, the less energy cost is, the lower energy efficiency can be achieved. Different from LEACH-A and HEED-A, the normal nodes in REELE choose the effective communication mode for themselves according to the reliable and energy-efficient communication strategy. A quantitative comparison can be made from Fig. 4 that REELE outperforms LEACH-A and HEED-A over $-42.2 \%$ and $-31.6 \%$ in energy efficiency, respectively.

In addition, we also draw another interesting observation from Fig. 4. The energy efficiency of LEACH-A is always stable, while it goes up rapidly after the time is greater than a certain value in HEED-A. This can be interpreted as follows. In LEACH-A, all nodes play a role as the leader for the same times, so the average energy consumption per bit is almost the same. However, HEED-A primarily consider the residual energy of the nodes, thus a closer node contends for the leader with a bigger probability at the start, while the probability of a farther node to become the leader raises as the residual energy decreases gradually over time, resulting in the increase of energy efficiency.

Fig. 5 compares the amount of energy spent by the leader per round in three algorithms. To show the results clearly, we present the results on LEACH-A and HEED-A in Fig. 5(b) on a small scale. From Fig. 5(a), we can see that the energy consumption by the leader per round in REELE is rather less than that in HEED-A and LEACH-A. The reason is two-fold. Firstly, the burden of the leader is relieved by using the novel communication strategy in REELE. Secondly, the node with the lowest total energy consumption is elected as the leader. 


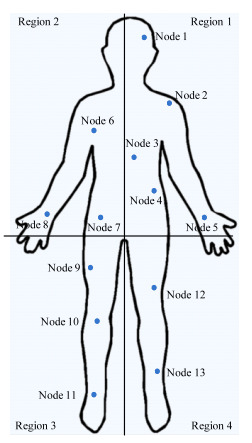

Fig. 1. The deployment of sensors

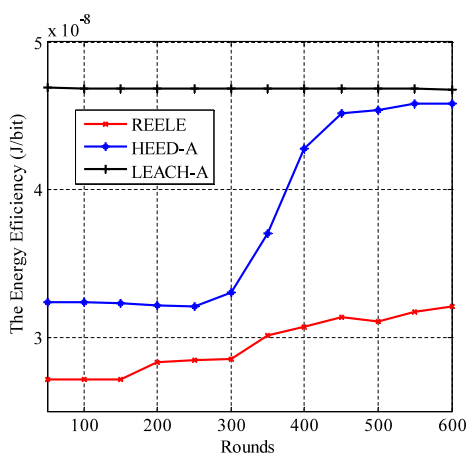

Fig. 4. The energy efficiency over time
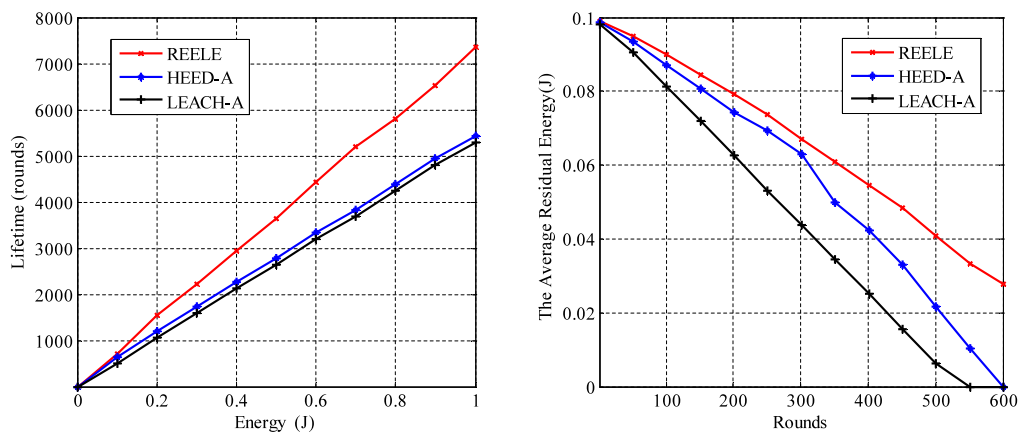

Fig. 2. The network lifetime over initial energy Fig. 3. The average residual energy over time

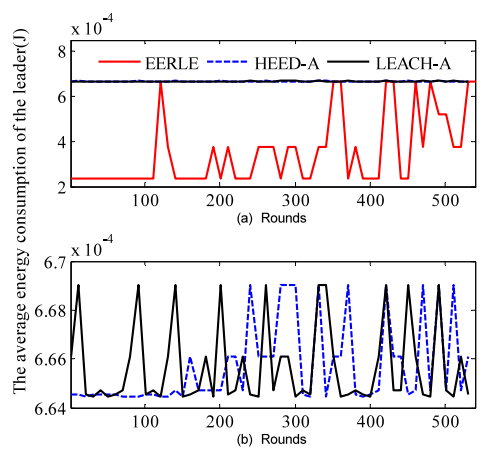

Fig. 5. The energy consumption of the leader

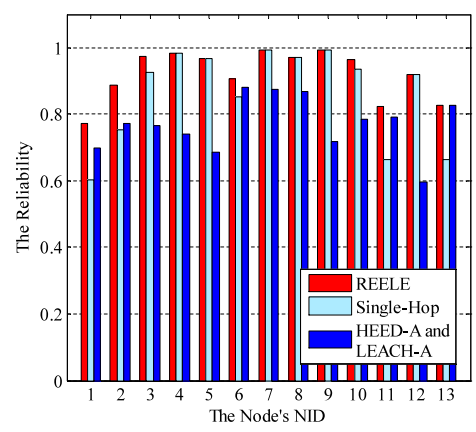

Fig. 6. The reliability of each node
In addition, a similar observation to that made for energy efficiency in Fig. 4 can be made from the Fig. 5(b) that the energy consumption of the leader in LEACH-A fluctuates regularly and in HEED-A it keeps low at the beginning and its expected value increase over time.

3) Reliability: In order to evaluate the network reliability, in Fig. 6 we show the reliability of each node in REELE. Compared with the single hop communication, the use of the reliable and energy-efficient communication strategy in REELE allows a remarkable performance enhancement for the far nodes as well as a good performance guarantee for the close nodes. However, in HEED-A and LEACH-A, the reliability of the close nodes is deteriorated substantially although that of the far nodes is improved slightly.

\section{CONCLUSION}

In this paper, we have studied a reliable and energy-efficient leader election scheme by partitioning a WBAN into regions. Based on the reliability analysis, we have proposed an effective communication strategy and further derived the total energy consumption model of a region. As the core of our work, a leader election algorithm, REELE, has been designed. In REELE, we jointly consider the impact of the reliability and residual energy of a node and total energy consumption on network performance. Extensive simulation results demonstrate the effectiveness and efficiency of REELE in prolonging network time and enhancing the reliability, which indicates the applicability of REELE to WBANs.

\section{REFERENCES}

[1] S. Movassaghi, M. Abolhasan, and et al., "Wireless body area networks: A survey," IEEE Communications Surveys and Tutorials, vol. 16, no. 3, pp. 1658-1686, 2014.

[2] V. Shankar, A. Natarajan, and et al., "Energy-efficient protocols for wireless communication in biosensor networks," in IEEE International Symposium on Personal, Indoor and Mobile Radio Communication (PIMRC), pp. 114-118, 2001.

[3] W. B. Heinzelman, A. P. Chandrakasan, and et al., "An applicationspecific protocol architecture for wireless microsensor networks," IEEE Transaction Wireless Communication, vol. 1, no. 4, pp. 660-670, 2002.

[4] O. Younis and S. Fahmy, "Heed: a hybrid, energy-efficient, distributed clustering approach for ad hoc sensor networks," IEEE Transaction on Mobile Computing, vol. 3, no. 4, pp. 366-379, 2004.

[5] A. Fort, J. Ryckaert, and et al., "Ultra-wideband channel model for communication around the human body," IEEE Journal on Selected Areas in Communication (JSAC), vol. 24, no. 4, pp. 927-933, 2006.

[6] J. Ding, E. Dutkiewicz, X. Huang, and G. Fang, "Energy-efficient cooperative relay selection for uwb based body area networks," in IEEE International Confer. on Ultra-Wideband (ICUWB), pp. 97-102, 2013.

[7] X. Huang, H. Shan, and X. Shen, "On energy efficiency of cooperative communications in wireless body area network," in IEEE Wireless Commun. and Network. Confer. (WCNC), pp. 1097-1101, IEEE, 2011.

[8] R. D'Errico, R. Rosini, and M. Maman, "A performance evaluation of cooperative schemes for on-body area networks based on measured timevariant channels," in IEEE International Conference on Communication (ICC), pp. 1-5, 2011.

[9] A. Astrin and et al., "IEEE standard for local and metropolitan area networks - part 15.6: Wireless body area networks," pp. 1-271, 2012.

[10] E. Reusens, W. Joseph, and et al., "Characterization of on-body communication channel and energy efficient topology design for wireless body area networks," IEEE Transaction on Information Technology in Biomedicine, vol. 13, no. 6, pp. 933-945, 2009. 